\title{
Electro-optical measurement of sub-ps structures in low charge electron bunches
}

\author{
F. Müller, ${ }^{*}$ P. Peier, and V. Schlott \\ PSI, Villigen, Switzerland \\ B. Steffen \\ DESY, Hamburg, Germany \\ T. Feurer \\ Institute of Applied Physics, University of Bern, Bern, Switzerland \\ P. Kuske \\ $H Z B$, Berlin, Germany \\ (Received 23 January 2012; published 12 July 2012)
}

\begin{abstract}
Electro-optical detection of $\mathrm{THz}$ coherent synchrotron radiation is a nondestructive method for measuring subpicosecond electron bunches or subpicosecond substructures on otherwise longer electron bunches. With a new diagnostic setup at the Swiss Light Source, which combines an amplified Yb fiber laser and a suitable GaP crystal, we demonstrate sampling as well as spectrally resolved single-shot measurements of sliced electron bunches containing as little as a few $\mathrm{pC}$ of charge. The single-shot measurements not only allow for a precise electric field characterization but also for a detailed analysis of the timing jitter between the electron bunch and the synchronized $\mathrm{Yb}$ fiber laser. The measurements of subsequent turns in the storage ring show distinct deviations from the simulations and we find strong indications that this discrepancy is caused by radiation loss through coherent synchrotron radiation itself, which is not included in many of today's simulation codes.
\end{abstract}

DOI: 10.1103/PhysRevSTAB.15.070701

PACS numbers: 78.20.Jq, 07.85.Qe, 41.60.Ap

\section{INTRODUCTION}

In a number of today's and upcoming accelerator facilities, the length of the electron bunches accelerated approaches picosecond to subpicosecond time scales. Moreover, several storage ring facilities operate in a standard mode and use ultrafast lasers to impose a subpicosecond charge distribution onto a several tens of picosecond long electron bunch. The latter technique is labeled electron bunch slicing [1]. The strong electric field of the laser pulse (up to $10^{9} \mathrm{~V} / \mathrm{m}$ ) modulates the energy of the electrons in a slice with a length comparable to the duration of the laser pulse. After a dispersive section this energy modulation is transferred into a longitudinal electron density modulation which then emits sub-ps hard x-ray pulses in a subsequent undulator beam line.

The longitudinal structure of such electron bunches can be indirectly analyzed by measuring either their Coulomb near-field or the coherent radiation emitted by them. In both cases the detection unit must have $\mathrm{THz}$ bandwidths which is why electro-optical (EO) techniques are

\footnotetext{
*Also at Institute of Applied Physics, University of Bern, Bern, Switzerland.

Published by the American Physical Society under the terms of the Creative Commons Attribution 3.0 License. Further distribution of this work must maintain attribution to the author(s) and the published article's title, journal citation, and DOI.
}

frequently used [2]. While the Coulomb field is always present, coherent radiation is emitted only in special geometries, for example when the electron bunches propagate through a bending magnet, a thin metal foil (transition radiation), or a slit or a hole in a metal (diffraction radiation). It was shown that the Coulomb field of sub-ps electron bunch structures with some $100 \mathrm{pC}$ charge can be measured with about 200 fs resolution by the spectral decoding technique $[3,4]$. Also, measuring the coherent transition radiation has proven to be a suitable method to analyze the temporal profiles of electron bunches [5]. A drawback of both methods is that they require a crystal or a metal foil close to or even in the path of the electrons and, thus, can either trigger wakefields or distort the electron bunches otherwise. An almost completely nondestructive method is to measure the coherent synchrotron radiation (CSR) emitted in a bending magnet. In the past the spectral content of CSR has been analyzed [6] and recently its time structure has been measured with electro-optic techniques [7].

Here, we present first scanning and single-shot EO measurements using a $\mathrm{Yb}$ fiber laser in combination with a $\mathrm{GaP}$ crystal. As the phase matching condition for $\mathrm{Yb}$ laser pulses in $\mathrm{GaP}$ is met almost perfectly (much better than for Ti:Sa lasers) the crystal thickness can be large leading to a very sensitive detection unit. With such a setup it was possible to perform first time domain measurements of sliced electron bunches at the Swiss Light Source (SLS) containing a charge of only $3.3 \mathrm{pC}$. Moreover, by observing 
the sliced electron distribution over four turns of the SLS storage ring we found a strong indication for an additional loss mechanism usually not included in most of today's simulation codes, which most likely is CSR itself.

\section{FUNDAMENTALS}

\section{A. Electron bunch slicing at the SLS}

The slicing setup at the SLS is used to generate sub-ps hard x-ray pulses mostly for pump-probe experiments [8]. It consists of a modulator (wiggler) for energy modulation, chicane dipoles for pulse separation, and a radiator (invacuum undulator). In the wiggler a strong laser pulse overlaps with the copropagating electron bunches and induces an energy exchange between the laser pulse and the electron bunch. Depending on the relative phase between the two pulses, the electrons can either gain or lose energy. The magnetic chicane between modulator and radiator induces a spatial $( \pm 2 \mathrm{~mm})$ and angular $( \pm 0.5 \mathrm{mrad})$ horizontal offset of the sliced beam at the radiator center which is used to separate the fs x-ray pulse from the ps background. Additionally, the chicane is a dispersive element which transforms the pure energy modulation of the electrons into a longitudinal density modulation. The longitudinal charge density modulation is of bipolar shape and a simulated charge distribution is shown in Fig. 1. One hump contains the electrons which gained energy whereas the other contains electrons that lost part of their energy. Both humps are separated by a dip from which the modulated electrons have been removed. X-ray diffraction measurements on the phonon dynamics of photo-excited bismuth are consistent with an effective $\mathrm{x}$-ray pulse duration and, thus, the full width at half maximum (FWHM) of the humps is $(140 \pm 30)$ fs [9].

For a comprehensive description of the slicing experiment at the SLS see Refs. [9,10]. Briefly, the slicing laser is a regenerative Ti:Sa amplifier with a pulse duration of $70 \mathrm{fs}$ FWHM, a repetition rate of $2 \mathrm{kHz}$, and a maximum pulse energy of $2.5 \mathrm{~mJ}$. The interaction between the laser and the electron charge distribution was simulated with $N$ particles within a time window of $\Delta T$ which can be chosen

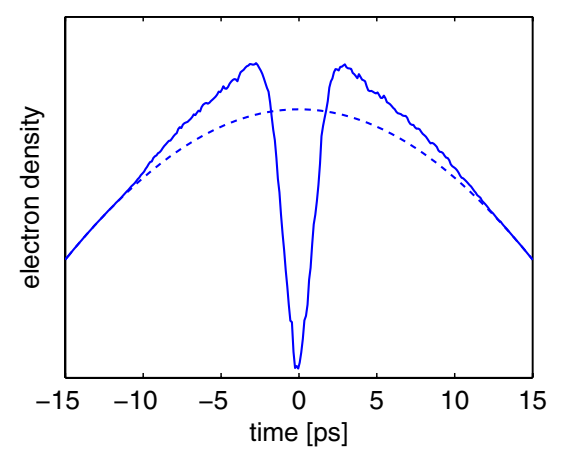

FIG. 1. Simulated charge distribution of the sliced electron bunch as a function of time immediately after slicing. arbitrarily as long as it is much shorter than the unmodulated electron bunch of duration $\sigma_{t}$ and longer than the laser pulse duration. The charge in this time window is given by $I_{\text {peak }} \Delta T$, with the peak current given by $I_{\text {peak }}=$ $Q_{\text {bunch }} /\left(\sqrt{2 \pi} \sigma_{t}\right)$ and the total bunch charge $Q_{\text {bunch }}=$ $I_{\text {bunch }} T_{\text {rev }}$. With the revolution time $T_{\text {rev }}=0.96 \mu \mathrm{s}$, $I_{\text {bunch }}=4 \mathrm{~mA}, \sigma_{t}=36 \mathrm{ps}(85 \mathrm{ps}$ FWHM $)$, and $\Delta T=$ $212 \mathrm{fs}$, we obtain a charge of $9.3 \mathrm{pC}$. According to simulations, a laser pulse energy of $2 \mathrm{~mJ}$ at the interaction region provides a maximum energy modulation of $1 \%$ $(24 \mathrm{MeV})$ [11]. In order to further simulate the redistribution of the sliced charge density upon propagation in the storage ring we use the particle tracking code TRACY-2 [12,13]. The basic idea of this tracking method is to split the electron distribution in three groups which can be tracked independently and finally superimposed. The first group contains all electrons from the 85 ps long core bunch which have not been modulated (energy spread $\frac{\Delta E}{E} \sim 8.7 \times 10^{-4}$ ). The second group contains the modulated electrons which have, due to the energy exchange with the laser, a higher energy spread of $\frac{\Delta E}{E} \sim 8.0 \times 10^{-3}$. The third group describes the dip, where electrons have been extracted from the core bunch, through a net positive charge or a positron distribution having the same energy spread as the core electrons. Immediately after modulation the sum of the second and the third charge distributions is zero. Then the two distributions separate in time and, because of the higher energy spread, the sliced electron distribution broadens much faster than the positron distribution. The charge of the resulting central dip is given by the number of particles for which the sum is $<0$, i.e., where the current is smaller compared to an unmodulated bunch, normalized to the number $N$ of all particles in the time window $\Delta T$ and multiplied by the charge in this window. For a laser pulse energy of $2 \mathrm{~mJ}$ we obtain $3.3 \mathrm{pC}$ for the hole charge independent of position, since the longitudinal drift only stretches but does not redistribute the charges. Thus, the slicing efficiency for the hole, given as the ratio of hole charge to bunch charge, amounts to $8.25 \times 10^{-4}$ which is roughly the ratio of laser pulse duration to bunch length (70 fs/85 ps).

For the remainder of this contribution all simulations as well as measurements refer to the infrared (IR) beam line of the SLS [14] which is located about three quarters of a round trip after the slicing experiment. Turn 0 identifies an electron bunch passing the IR beam line directly after slicing, turn 1 an electron bunch that has revolved around the storage ring one additional full turn, etc. The modulation at the IR beam line is already somewhat longer than directly after interaction with the laser and it broadens further with every additional turn. The simulated longitudinal charge distribution of only the sliced electron and positron bunches is shown in Fig. 2 [15]; for clarity the original 85 ps long electron bunch is not shown. The FWHM of the dip at turn 0 is $750 \mathrm{fs}$ and it broadens by about 1 ps per revolution. 


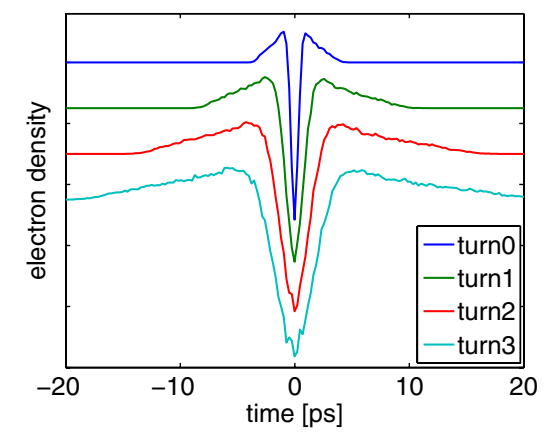

FIG. 2. Simulated charge distribution of the sliced bunches as a function of time from turn 0 to turn 3 . The curves are shifted vertically for better visibility.

The simulated synchrotron spectrum emitted by the sliced bunch at the IR beam line bending magnet (turn 0) is depicted in Fig. 3. The core bunch generates coherent and incoherent synchrotron radiation. The coherent part, however, is at very long wavelengths and is suppressed by the SLS vacuum chamber (indicated by the hatched area in Fig. 3). The sliced particles radiate coherently at wavelengths comparable to the sliced bunch length and, therefore, the spectrum is coherently enhanced in the THz range. The spectrum changes from turn to turn because the charge distribution is modified through nondispersive effects.

Integrating the coherent part of the $\mathrm{THz}$ spectrum indicates that not only does the spectral shape change but also the overall energy radiated from the bunch. Table I shows the energy of the coherently emitted synchrotron radiation for increasing number of turns. The radiated energy decreases to about $14 \mathrm{pJ}$ in only 4 turns.

\section{Beam transfer line}

From the bending magnet at the IR beam line the $\mathrm{THz}$ radiation is transported to the detection unit through an evacuated transfer line which is equipped with three reflective relay imaging optics. Because of several limiting apertures and the finite size of the mirrors used, the transfer

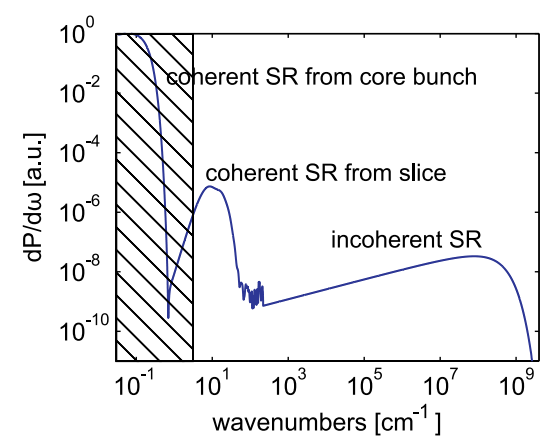

FIG. 3. Simulated spectrum of the synchrotron radiation emitted from the sliced electron bunch at the IR beam line. Spectral components within the hatched area are not transported by the beam line.
TABLE I. Energy per pulse of the CSR at the bending magnet of the IR beam line for several turns after slicing.

\begin{tabular}{cc}
\hline \hline Turn & Energy [pJ] \\
\hline 0 & 138.9 \\
1 & 40.7 \\
2 & 22.4 \\
3 & 14.3 \\
\hline \hline
\end{tabular}

line exhibits a high-pass characteristic with the cutoff frequency close to the frequency range of interest. It is therefore crucial to know the transfer function of the beam transfer line. The transfer function was simulated with SRW $[16,17]$ and measured experimentally. To that end we assume that the incoherent synchrotron radiation emitted by the electron bunch (slicing laser off) is constant within the frequency range of interest and that the intensity transmission coefficient $T(\omega)$ follows the transmitted spectrum, which we measured with a Martin Puplett interferometer. In addition we assume that $T(\omega)$ approaches 1 for frequencies higher than $1 \mathrm{THz}$. Figure 4 shows a fit to the measured (blue curve) and the simulated (green curve) transfer function versus wave number and a good agreement is found. Both curves indicate a strong suppression of all wavelengths longer than about $1 \mathrm{~mm}$.

For reasons of causality such an amplitude modulation evokes a spectral phase modulation $\phi(\omega)$, which is difficult to measure but can be estimated by means of KramersKronig relations $[18,19]$. The complex field transmission coefficient is determined by

$$
t(\omega)=\sqrt{T(\omega)} \mathrm{e}^{i \phi(\omega)} .
$$

Rewriting Eq. (1) in the form $\ln [t(\omega)]=$ $1 / 2 \ln [T(\omega)]+i \phi(\omega)$ allows one to use the Hilbert transform to reconstruct the unknown phase $\phi(\omega)$ from $1 / 2 \ln [T(\omega)]$. Note that $-\ln [T(\omega)]=\alpha(\omega) l$ yields the effective absorption coefficient $\alpha(\omega)$ multiplied by the length of the beam line $l$, which is shown in Fig. 5(a). Figure 5(b) shows the phase $\phi(\omega)-\omega l / c$, where the linear phase due to the drift length was subtracted. With

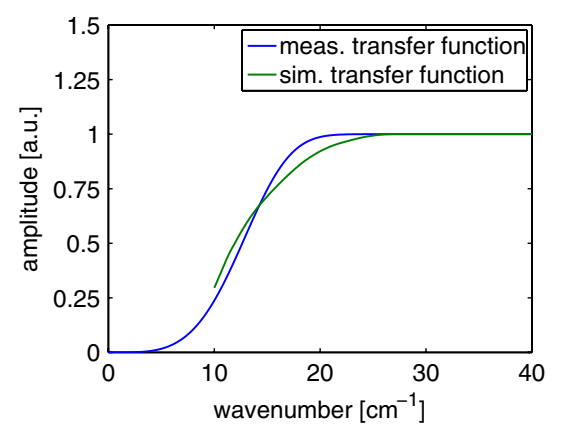

FIG. 4. The simulated (green curve) as well as a fit to the measured transfer function (blue curve) as a function of wave number. 

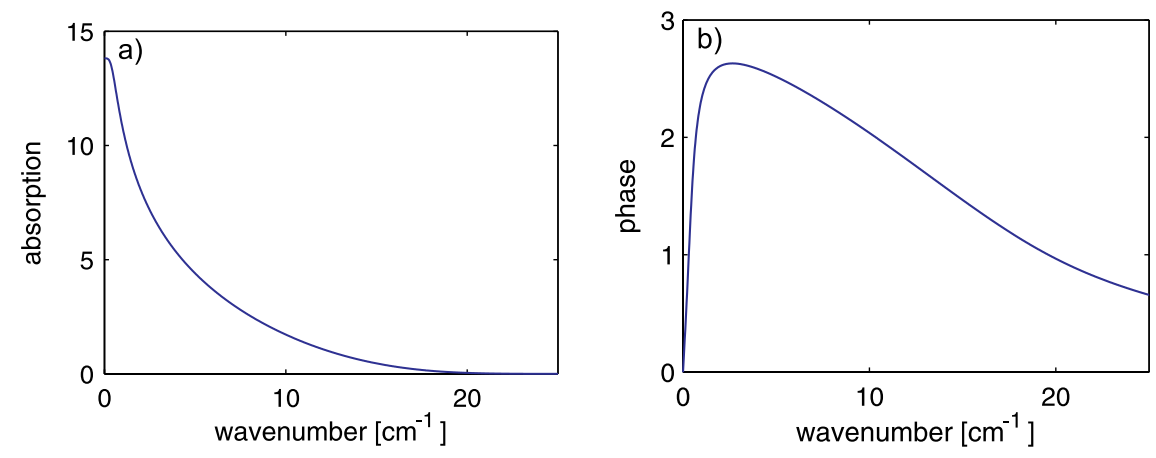

FIG. 5. (a) Absorption and (b) derived phase modulation as a function of wave number.

the amplitude and phase modulation we can calculate the expected temporal profile of the electric field at the position of the electro-optic detection unit.

\section{Electro-optical detection}

Electro-optically active crystals, like GaP, become birefringent in the presence of an electric field. THz electrooptical detection techniques exploit this effect to measure $\mathrm{THz}$ electric fields by sampling the change in induced birefringence with femtosecond laser pulses. For small electric fields (up to some $\mathrm{MV} / \mathrm{m}$ ), the EO effect is dominated by the Pockels effect and is linear with the $\mathrm{THz}$ electric field strength. Here, we use a GaP crystal oriented such that for normal incidence the electric field is in the (110)-plane and the $\mathrm{THz}$ and the pump fields are polarized along the $x$ axis. For this configuration the two orthogonally polarized components of the probe laser undergo a relative phase retardation of

$$
\Gamma=\frac{\omega d}{c}\left(n_{1}-n_{2}\right)=\frac{\omega d}{c} n_{0}^{3} r_{41} E_{\mathrm{THz}} G(\omega, f, d),
$$

where $\omega$ denotes the laser frequency, $d$ the thickness of the EO crystal, $n_{1}$ and $n_{2}$ the refractive indices along the principal axes, $n_{0}$ the refractive index of the isotropic crystal without external electric field, $r_{41}$ the EO coefficient, and $E_{\mathrm{THz}}$ the applied electric field. The EO efficiency

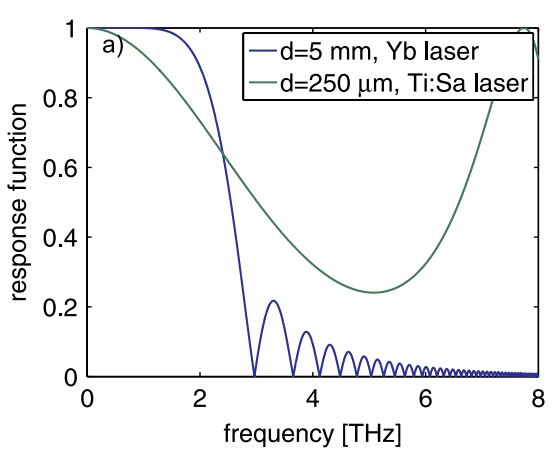

can be characterized by the response function $G(\omega, f, d)$ [20] which depends on the laser frequency $\omega$, the $\mathrm{THz}$ frequency $f$, and the $\mathrm{GaP}$ crystal properties. The response function is displayed in Fig. 6(a). Because of the almost ideal phase matching conditions for the combination $\mathrm{Yb}$ laser and $\mathrm{GaP}$ crystal, the required bandwidth of approximately $2 \mathrm{THz}$ can be achieved with a $5 \mathrm{~mm}$ thick GaP crystal (blue curve). To obtain a similar bandwidth using a Ti:Sa laser (green curve) requires a much thinner crystal thickness of only $0.25 \mathrm{~mm}$ which in turn results in a much lower sensitivity as can be seen in Fig. 6(b) where the two response functions are compared on an absolute scale.

In order to detect the modified state of polarization after the EO crystal, it has to be converted to an amplitude modulation, ideally linearly proportional to the applied $\mathrm{THz}$ electric field. This is achieved by a combination of wave plates and polarizers. In the following sections, we present two different types of arrangements, namely a scanning-type EO sampling technique (EOS) and a spectrally resolved EO decoding technique (EOSD), as shown in Fig. 7. The EOS method [Fig. 7(a)] uses a short laser pulse ( $\approx 150 \mathrm{fs}$ ) which propagates in parallel with the $\mathrm{THz}$ electric field through the crystal. Its relative delay is scanned with respect to the $\mathrm{THz}$ pulse in order to sample the entire temporal profile of the THz electric field. Since the profile is not measured in a single shot, this technique is

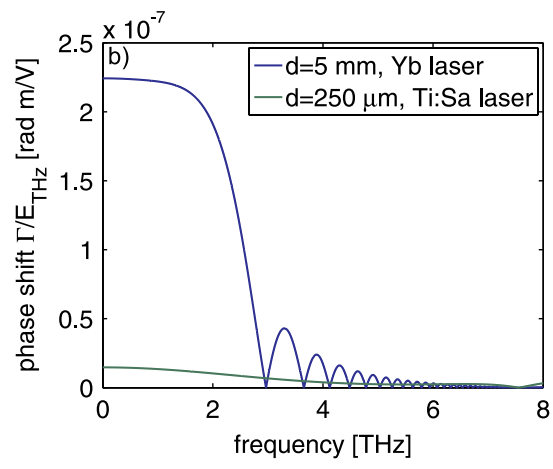

FIG. 6. (a) Response function for Yb laser pulses in a $5 \mathrm{~mm}$ (blue curve) and Ti:Sa laser pulses in a $250 \mu \mathrm{m}$ thick GaP crystal (green curve). (b) The phase shift, i.e., the overall efficiency, is almost 20 times higher for Yb laser (blue curve) than for Ti:Sa laser (green curve). 
sensitive to intensity fluctuations of the $\mathrm{THz}$ radiation and the $\mathrm{Yb}$ fiber laser as well as to the relative arrival time jitter between the two.

Conversely, EOSD [Fig. 7(b)] is a single-shot technique where the probe laser pulse is stretched in a dispersive material or a stretcher to a duration longer than the expected $\mathrm{THz}$ electric field. With the known timeto-frequency mapping, the temporal shape of the $\mathrm{THz}$ electric field can simply be measured with a spectrometer. For a detailed analysis of the signal a few subtleties of the frequency mixing process have to be considered. Specifically, mixing the $\mathrm{Yb}$ laser and the $\mathrm{THz}$ electric field may lead to signal distortions for short $\mathrm{THz}$ pulses and/or
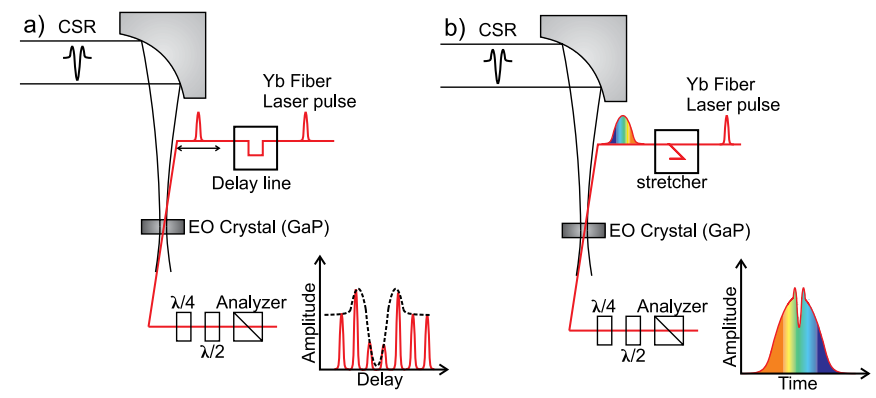

FIG. 7. Schematic of (a) the electro-optic sampling (EOS) and (b) the spectrally resolved electro-optic detection (EOSD) setup. strongly stretched laser pulses [21] and, thus, the bandwidth-limited duration of the Yb laser pulses should be much shorter than the oscillation period of the $\mathrm{THz}$ electric field to be measured. Moreover, the induced phase retardation $\Gamma$ [Eq. (2)] depends on the laser frequency $\omega$ and the sensitivity changes by about $10 \%$ over the laser spectrum between 1.0 and $1.1 \mu \mathrm{m}$. In EOSD experiments this frequency dependent sensitivity [see Fig. 8(a)] causes modifications to the measured spectral image of temporal $\mathrm{THz}$ electric field. If we compare the same $\mathrm{THz}$ electric field measured at different relative arrival times between the $\mathrm{THz}$ pulse and the stretched probe pulse this effect becomes visible, as shown in Fig. 8(b). The minimum as well as the maximum amplitude decrease with increasing time delay. For a more comprehensive description of EO detection techniques, theory, and the simulations see also Refs. [4,22].

\section{Summary}

Combining the results discussed above allows simulating the expected $\mathrm{THz}$ electric field at the detection setup as well as the expected EO signals for both detection units. Figure 9(a) shows the turn $0 \mathrm{THz}$ electric field emitted from the bending magnet of the IR beam line (green curve) and the THz electric field after the beam transfer line (blue curve). While the amplitude modulation leads to a visible
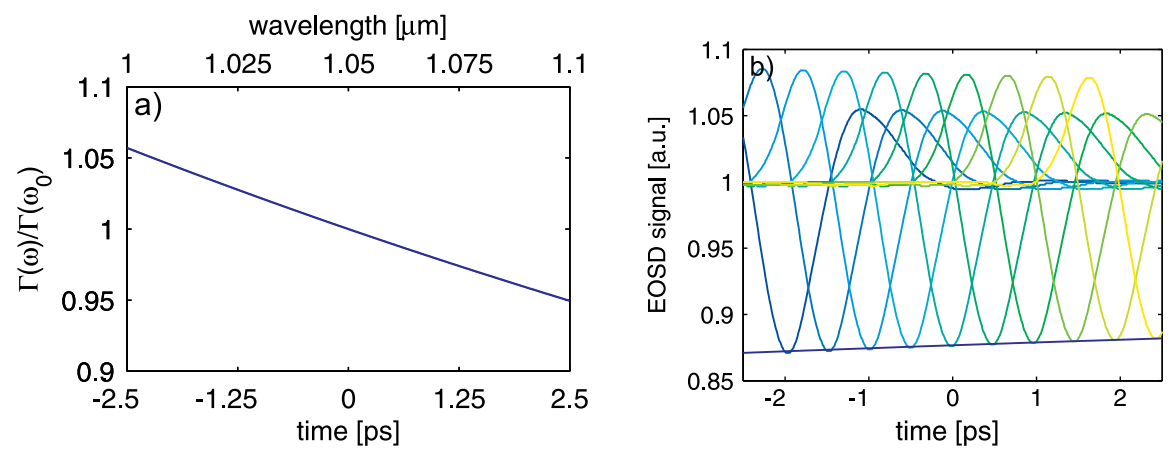

FIG. 8. (a) In EOSD experiments the probe wavelength dependent sensitivity translates into a delay dependent sensitivity. (b) Simulated EOSD signals for ten different relative arrival times.
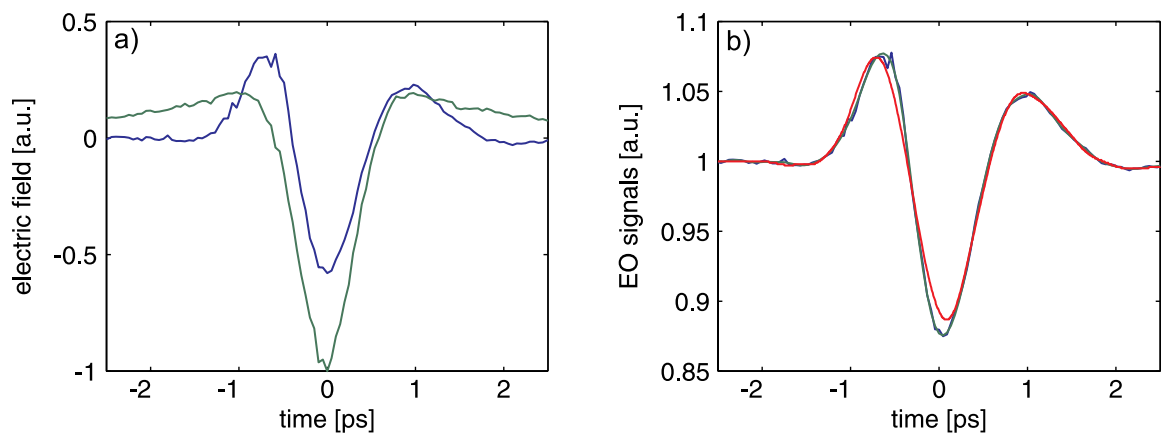

FIG. 9. (a) THz field emitted at turn 0 (green curve) in comparison to the expected electric field distribution after the beam transfer line (blue curve). (b) The corresponding simulated EOS (green curve) and EOSD (red curve) signals. 
loss of low frequency contributions, the phase modulation leads to an almost constant phase offset between the carrier and the envelope resulting in a slightly asymmetric trace.

Figure 9(b) shows the simulated EOS (green curve) and EOSD (red curve) signals in comparison to the expected electric field distribution (blue curve). While the sampled EOS signal overlaps almost perfectly with the THz electric field, the EOSD signal shows some minor distortions which are due to the frequency mixing process. However, the changes are minor and may be neglected for the measurements reported hereafter.

\section{EXPERIMENTAL DETAILS}

The SLS circulates a $2.4 \mathrm{GeV}$ electron beam which emits synchrotron radiation in a $1.4 \mathrm{~T}$ dipole magnet at the IR beam line. The radiation is collected by a slotted mirror over an angle of $61 \mathrm{mrad}$ (corresponding to a source length of $350 \mathrm{~mm}$ ) and transported over $9 \mathrm{~m}$ by three 1-to-1 imaging optics to the experimental area outside the accelerator bunker. At the EO setup the CSR is imaged by a $250 \mathrm{~mm}$ off-axis paraboloid mirror into a $5 \mathrm{~mm}$ thick GaP crystal (Moltech, Berlin) leading to a spot size of 5-10 mm in diameter. The EO probe laser is a home-built amplified ytterbium fiber laser system [23] synchronized to the SLS radio frequency (rf) master clock. It delivers $10 \mathrm{~nJ}$ pulses at $1 \mathrm{MHz}$ repetition rate with a bandwidth of about $120 \mathrm{~nm}$ centered at $1050 \mathrm{~nm}$ resulting in a bandwidth-limited pulse duration of approximately $30 \mathrm{fs}$. Since the slicing of the electron bunches is done with an independently synchronized Ti:Sa laser, the stability and the relative timing jitter between the two lasers play a decisive role for $\mathrm{THz}$ pulse length measurements. The timing jitter was measured to approximately $50 \mathrm{fs}$ (rms) by an independent cross correlation experiment [23].

For the EOS measurements the $\mathrm{Yb}$ fiber laser pulses were stretched to about 150 fs (FWHM) in a grating compressor. In the EOSD experiments the probe pulses were stretched to $4.7 \mathrm{ps}$ (FWHM) in order to ensure sufficient temporal overlap even in the presence of arrival time jitter. In both cases the pulses were sent via a polarizer rotated by a small angle through the GaP crystal. After passing the crystal the pulses were directed through a quarter wave plate, a half wave plate, and an analyzing polarizer to an optical fiber. All measurements were done with the half wave plate $2^{\circ}-4^{\circ}$ away from crossed polarization settings to ensure good linearity in the measured signals. For the EOS experiments the pulses were detected by an InGaAs photodiode (ET-3010, Electro-Optics Technology, Inc.) and the readout and averaging was done by an oscilloscope. For EOSD measurements a home-built spectrometer equipped with an InGaAs camera (Andor iDus490-1.7) was used.

\section{RESULTS}

\section{A. Zero turn}

Figure 10 shows an EOS and several single-shot EOSD measurements of the THz CSR emitted after turn 0 . For the EOS measurements the $\mathrm{Yb}$ laser is delayed in increments of $100 \mathrm{fs}$ and each data point represents an average over 100 individual acquisitions. The central dip in Fig. 10(a) has a FWHM of $1.2 \mathrm{ps}$. Already the relatively large fluctuations of consecutive acquisitions at a fixed time delay indicate a significant timing jitter between the sampling laser and the sliced electron bunch.

This presumption was then verified through recording many consecutive single-shot EOSD measurements; a selection of them is shown in Fig. 10(b). The FWHM of the central dip was determined from a fit of two Gaussians with amplitudes of opposite signs to the experimental data and amounts to $(880 \pm 6)$ fs. This value is significantly smaller than the value extracted from the EOS measurements and the difference is attributed to the arrival time jitter. Moreover, the EOSD measurements can be used to precisely determine the arrival time jitter and also its statistics. Figure 11(a) shows a histogram of the arrival time jitter for a sequence of 500 measurements. The histogram (blue curve) of the arrival time jitter is fitted to a Gaussian distribution (green curve) and we find a standard deviation of $330 \mathrm{fs}$ rms. As the measurements are taken with a constant sampling frequency of approximately

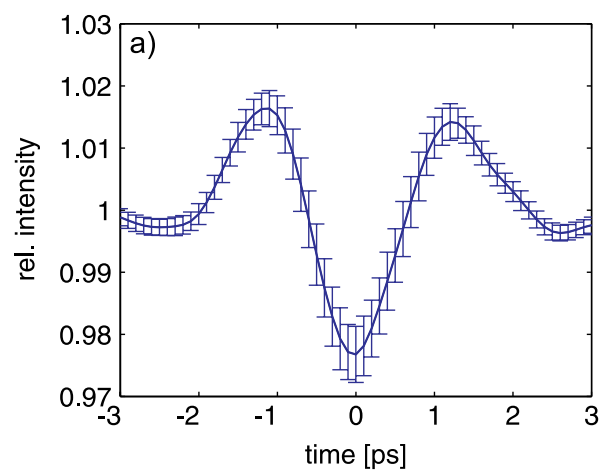

FIG. 10. (a) EOS measurement and (b) several consecutive single-shot EOSD measurements (green curves) together with a fit to two Gaussian-shaped distributions (blue curves). The curves are shifted vertically for better visibility. 

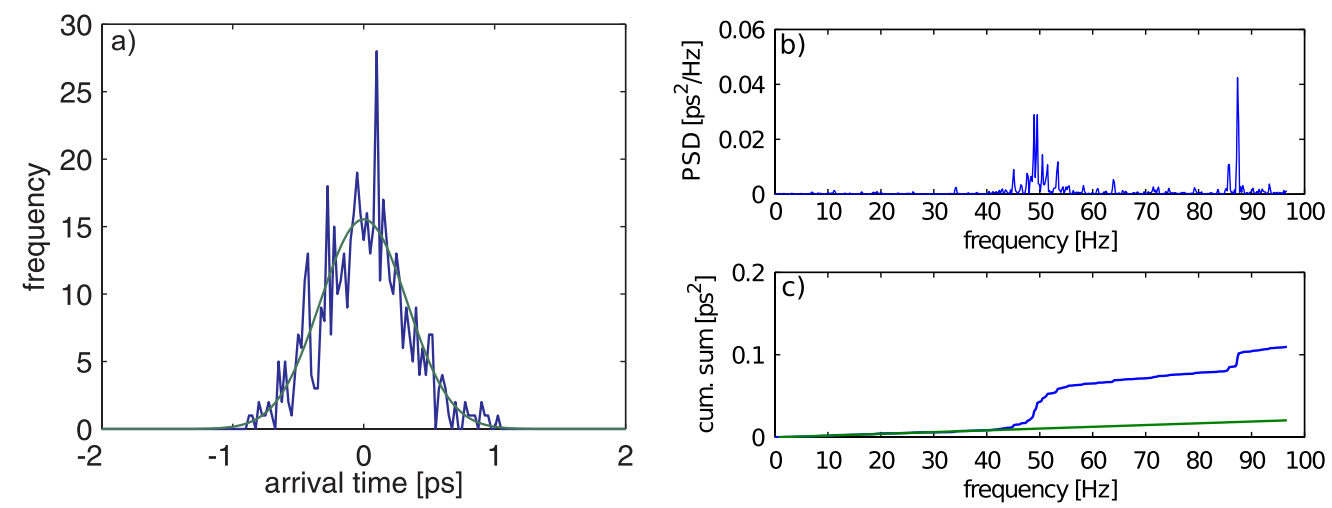

FIG. 11. (a) Histogram of the arrival time jitter (blue line) and a fitted Gaussian distribution with a rms width of 330 fs (green curve). (b) Power spectral density of the arrival time jitter. (c) The cumulative sum (blue curve) indicates the jitter contribution at different frequencies and the uncorrelated jitter of $145 \mathrm{fs}$ (green curve) is the minimal resolution of the setup.

$200 \mathrm{~Hz}$, the spectral distribution of the timing jitter can be studied up to the Nyquist frequency of $100 \mathrm{~Hz}$. The power spectral density normalized to the square of the standard deviation [Fig. 11(b)] relates the arrival time jitter contributions at different frequencies. The cumulative sum [Fig. 11(c)] allows determining the arrival time jitter contribution at a certain frequency. Assuming that the two steps at 50 and $90 \mathrm{~Hz}$ are due to external sources the remaining uncorrelated arrival time jitter grows linearly as indicated by the green curve and adds up to $145 \mathrm{fs}$. The uncorrelated arrival time jitter determines the resolution of the arrival time measurement. Thus, the resulting arrival time jitter, which is dominated by contributions at 50 and $90 \mathrm{~Hz}$, could be determined to $295 \mathrm{fs}$ and it leads to a significant and measurable distortion of the EO sampling results. So far, a source for this comparatively large timing jitter at the IR beam line could not be identified, however, amplitude and phase jitter of the storage ring $\mathrm{rf}$ and timing distribution as well as mechanical vibrations in the IR beam line are major suspects.

As the arrival time in every single measurement is known, the jitter can be corrected before averaging. Such averaged measurements can then be compared to particle tracking simulations if the spectral filter function of the

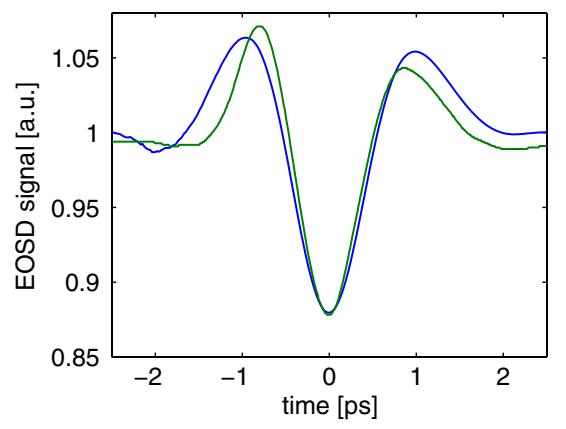

FIG. 12. EOSD measurement (blue) compared to the simulation (green) filtered with the transfer function of the IR beam line. beam transfer line and the frequency mixing process are taken into account. Figure 12 shows the measured signal for turn 0 (blue curve) which is in good agreement with the simulated signal (green curve). From the modulation depth and the incoming probe laser intensity, we calculate the maximum phase shift $\Gamma=-0.57 \mathrm{deg}$ from $I_{1}=$ $I_{0} / 2[1-\cos (\Gamma-4 \theta)]$ with $\theta=2$ deg. Using Eq. (2) results in a field strength of $E_{\mathrm{THz}}=3.6 \times 10^{4} \mathrm{~V} / \mathrm{m}$ and considering the Fresnel loss at the air/GaP interface yields the maximum field $E_{\mathrm{THz}}=7.9 \times 10^{4} \mathrm{~V} / \mathrm{m}$.

In addition, the predicted wavelength dependent sensitivity in EOSD experiments is confirmed as can be seen in Fig. 13. The measurements for ten different arrival times between the stretched laser pulse and $\mathrm{THz}$ electric field show the same decrease as the simulated profiles shown in Fig. 8(b).

\section{B. Subsequent turns}

Because of the very high sensitivity of the setup it is possible to detect not only the $\mathrm{THz}$ CSR from turn 0 but also that from subsequent turns. Figure 14(a) shows EOS results for the first four turns and Fig. 14(b) EOSD measurements for the first two turns after slicing occurs. It can

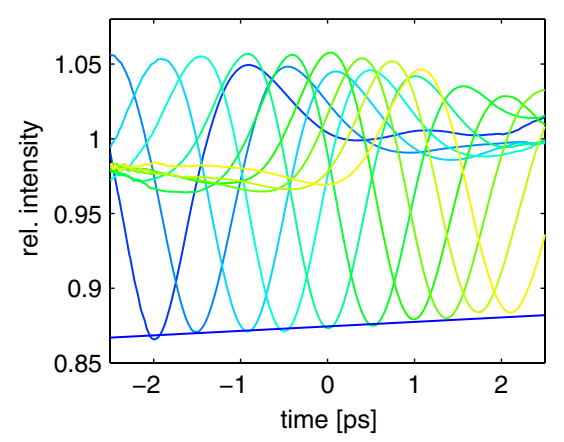

FIG. 13. Averaged EOSD measurements for ten different arrival times. 

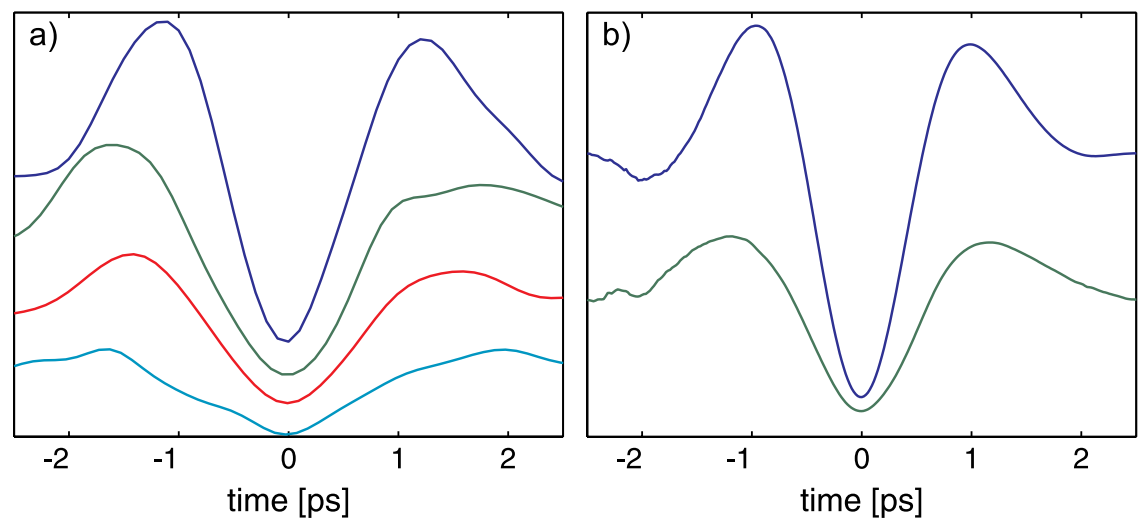

FIG. 14. (a) EOS measurements from turn 0 to turn 3. (b) Arrival time jitter corrected average of 500 EOSD measurements for turn 0 and turn 1 . The curves are shifted vertically for better visibility.

be seen that the modulation depth decreases and the width of the central dip increases with the number of turns.

The particle tracking code (TRACY) predicts a broadening of the central dip of about 1 ps per turn which is considerably larger than what is observed in both measurements. A similar discrepancy was already found when characterizing the spectrum of the CSR as a function of turns [6], showing the spectral width of the THz signal to be larger than expected. Such observations have also been made by other laboratories when examining the properties of their low alpha operation modes (e.g. ANKA [24] and BESSY II) as well as during the femto-slicing experiments at BESSY II [25]. In all cases, the spectral shift of the CSR spectrum towards lower frequencies with consecutive turns is significantly smaller than predicted by the simulations.

Several possible causes were evaluated, as, for example, variations of the slicing laser parameters, but they all predict an even faster broadening and had to be discarded. A likely explanation is that the simulation code TRACY does not include losses due to CSR from the sliced bunches. The integration of this effect in a particle tracking code based on the Vlasov-Fokker-Planck equation [26] results in a better agreement between simulation and ex-

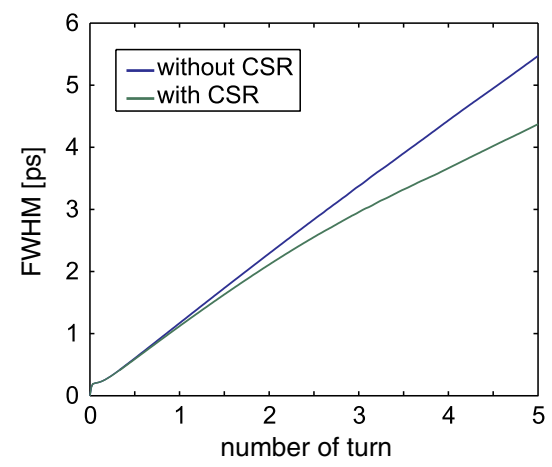

FIG. 15. Simulated FWHM of the central dip in the charge density distribution without (blue curves) and with (green curves) CSR losses as a function of the number of turns. perimental observations for both, the slicing experiment at the BESSY II [25] and the SLS storage ring. The simulations for the SLS predict that the effect of energy loss due to CSR is negligible for turn 0 , which is in good agreement with our experimental results. Analyzing the simulations indicates that the CSR wake leads to a slightly asymmetric and noticeably less broadened dip for higher slicing turns as shown in Fig. 15. The asymmetry together with the shorter pulse duration of the central dip translate into a spectrum which is shifted towards higher frequency components. This is in qualitative agreement with the spectral measurements for the SLS femto-slicing, which have been presented in Ref. [6], and the EO measurements presented here. Thus, it can be concluded that the impact of CSR losses is a possible reason for the experimentally observed slower broadening of the femto-sliced modulation from turn to turn, however, more experiments have to be done to verify this effect also quantitatively.

\section{CONCLUSION}

We presented EO measurements of sliced electron bunches using an $\mathrm{Yb}$ fiber laser in combination with a $\mathrm{GaP}$ crystal. Because of the superior phase matching it was possible to perform the first time domain measurements of the very weak subpicosecond longitudinal slicing substructure containing a charge of approximately $3.3 \mathrm{pC}$. When considering the transfer function of the SLS IR beam line as well as the EO frequency mixing process, the measured width of the longitudinal $\mathrm{THz}$ electric field profile for turn 0 agrees well with the simulations. After one or more revolutions the central dip of the slicing modulation is considerably smaller than the one predicted by the particle tracking simulation TRACY. The observed broadening of the modulation depth of the electron density might be explained by taking into account the CSR losses of the sliced bunch. Preliminary particle tracking simulation including CSR losses can explain the trend but up to now not the magnitude of the effect. More elaborate 
particle tracking simulations including CSR losses will be compared to the measurements in a future work.

\section{ACKNOWLEDGMENTS}

We acknowledge crucial support by P. Lerch, L. Quaroni, and H. Sigg for using the IR beam line, and by G. Ingold, S. Johnson, and P. Beaud for assistance with the slicing experiment. We would also like to acknowledge stimulating and fruitful discussions with S. Jamison. This work was funded in part by the Swiss National Science Foundation Grant No. 200021-112307 and T. F. acknowledges support by the NCCR MUST, research instrument of the Swiss National Science Foundation.

[1] A. A. Zholents and M. S. Zolotorev, Phys. Rev. Lett. 76, 912 (1996).

[2] S. P. Jamison, G. Berden, A. M. MacLeod, B. Steffen, P. J. Phillips, and W. A. Gillespie, in Proceedings of the 10th European Particle Accelerator Conference, Edinburgh, Scotland, 2006 (EPS-AG, Edinburgh, Scotland, 2006).

[3] I. Wilke, A. M. MacLeod, W. A. Gillespie, G. Berden, G. M. H. Knippels, and A. F. G. van der Meer, Phys. Rev. Lett. 88, 124801 (2002).

[4] B. Steffen, Ph.D. thesis, University of Hamburg, 2007.

[5] G. Berden, A. F. G. van der Meer, S. P. Jamison, B. Steffen, E.-A. Knabbe, B. Schmidt, P. Schmüser, A. M. MacLeod, P. J. Phillips, and W. A. Gillespie, in Proceedings of the 10th European Particle Accelerator Conference, Edinburgh, Scotland, 2006 (Ref. [2]).

[6] D. Abramsohn, Master's thesis, Technische Universitaet Berlin, 2009.

[7] I. Katayama, H. Shimosato, M. Bito, K. Furusawa, M. Adachi, M. Shimada, H. Zen, S. Kimura, N. Yamamoto, M. Hosaka, M. Katoh, and M. Ashida, Conference on Lasers and Electro-Optics (CLEO) San Jose, 2010.

[8] G. Ingold, P. Beaud, S. L. Johnson, V. Schlott, T. Schmidt, and A. Streun, PSI Technical Report, 2007.

[9] P. Beaud, S. L. Johnson, A. Streun, R. Abela, D. Abramsohn, D. Grolimund, F. Krasniqi, T. Schmidt,
V. Schlott, and G. Ingold, Phys. Rev. Lett. 99, 174801 (2007).

[10] D. Kalantaryan, G. Amatuni, V. Tsakanov, P. Beaud, G. Ingold, and A. Streun, in Proceedings of the 11th European Particle Accelerator Conference, Genoa, 2008 (EPS-AG, Genoa, Italy, 2008).

[11] P. Beaud (private communication).

[12] J. Bengtsson, TRACY-2 User's Manual, SLS Internal Document, PSI, Villigen, 1997.

[13] M. Boege, Paul Scherrer Institute Technical Report, 1999.

[14] http://www.psi.ch/sls/ir/ir.

[15] A. Streun (private communication).

[16] Synchrotron Radiation Workshop (SRW) [http://www.esrf .eu/Accelerators/Groups/InsertionDevices /Software/SRW].

[17] O. Chubar and P. Elleaume, in Proceedings of the 6th European Particle Accelerator Conference, Stockholm, 1998 (IOP, London, 1998).

[18] H. A. Kramers, Nature (London) 117, 775 (1926).

[19] R. de L. Kronig, J. Opt. Soc. Am. 12, 547 (1926).

[20] Q. Wu and X.-C. Zhang, Appl. Phys. Lett. 70, 1784 (1997).

[21] W. A. Gillespie, A. M. MacLeod, S. P. Jamison, and G. Berden, in Proceedings of the 11th European Particle Accelerator Conference, Genoa, 2008 (Ref. [10]).

[22] S. Casalbuoni, H. Schlarb, B. Schmidt, P. Schmüser, B. Steffen, and A. Winter, Phys. Rev. ST Accel. Beams 11, 072802 (2008).

[23] F. Müller, S. Hunziker, V. Schlott, B. Steffen, D. Treyer, and T. Feurer, in Proceedings of the 9th European Workshop on Beam Diagnostics and Instrumentation for Particle Accelerators DIPAC, Basel, 2009.

[24] N. Hiller, S. Hillenbrand, A. Hoffmann, E. Huttel, V. Judin, B. Kehrer, M. Klein, S. Marsching, A.-S. Müller, A. Plech, N. Smale, K. G. Sonnad, and P.F. Tavares, in Proceedings of the International Particle Accelerator Conference, Kyoto, Japan (ICR, Kyoto, 2010); W. A. Gillespie, A. M. MacLeod, S. P. Jamison, and G. Berden, in Proceedings of the 11th European Particle Accelerator Conference, Genoa, 2008 (Ref. [10]).

[25] P. Kuske (private communication).

[26] M. Venturini and R. Warnock, Phys. Rev. Lett. 89, 224802 (2002). 